\title{
L'innovation comme invention nécessaire dans tout processus d'acquisition. Former des praticiens de l'espagnol à l'école primaire
}

Isabelle Aliaga

\section{OpenEdition}

Journals

Édition électronique

URL : http://journals.openedition.org/trema/1452

DOI : 10.4000/trema. 1452

ISSN : 2107-0997

Éditeur

Faculté d'Éducation de l'université de Montpellier

\section{Édition imprimée}

Date de publication : 1 janvier 2003

Pagination : 143-160

ISSN : 1167-315X

\section{Référence électronique}

Isabelle Aliaga, «L'innovation comme invention nécessaire dans tout processus d'acquisition. Former des praticiens de l'espagnol à l'école primaire », Tréma [En ligne], 20-21 | 2003, mis en ligne le 01 avril 2003, consulté le 21 avril 2019. URL : http://journals.openedition.org/trema/1452 ; DOI : 10.4000/ trema.1452

Ce document a été généré automatiquement le 21 avril 2019

Trema 


\title{
L'innovation comme invention nécessaire dans tout processus d'acquisition. Former des praticiens de l'espagnol à l'école primaire
}

\author{
Isabelle Aliaga
}

\section{Introduction}

1 Ce numéro de Tremaest consacré à l'action innovante dans la formation des maîtres ; or, il semble bien que la définition la plus courante du concept d'innovation renvoie dans la plupart de nos dictionnaires ${ }^{1}$, au fait d'introduire quelque chose de nouveau, d'inconnu dans un ordre établi, dans un système déjà construit.

2 Je voudrais dire ici, en guise de préambule, que le dispositif de coopération-action auquel j'ai abouti dans mon travail de formatrice en espagnol pour les PE2 de l'IUFM de Montpellier, au bout de huit ans de réflexion, et qui a été retenu par la cellule innovation de l'IUFM de Montpellier comme un projet innovant, n'est en fait que le résultat d'un long parcours, de remises en questions et de réadaptations successives pour tenter de trouver enfin un dispositif de formation qui me permettrait d'atteindre mon objectif premier : la formation des enseignants d'espagnol à l'école primaire.

3 L'innovation m'a donc été dictée non par un désir d'originalité si je puis dire, mais par la nécessité urgente de former à partir des années 1990, de nouveaux maîtres du primaire à une nouvelle discipline : la langue étrangère et l'espagnol dans mon cas.

Aussi, avant d'examiner plus précisément les apports du dispositif de formation pour lequel j'ai reçu l'appellation d'action innovante, il me semble nécessaire dans un premier temps de retracer et d'analyser ici, le processus qui m'a amenée à construire précisément ce dispositif de coopération - action avec une école en sélectionnant un certain nombre de paramètres de formation à conserver, et cela pour répondre aux problèmes de la 
formation des maîtres du premier degré en espagnol et transmettre ou mutualiser ce que je pouvais connaitre de cet enseignement de l'espagnol à l'école.

4 Si lors de mon arrivée à l'IUFM de Montpellier, je connaissais l'enseignement pour les adolescents, comme professeur d'espagnol du secondaire, j'étais tout de même assez ignorante ${ }^{2}$ des données psychologiques cognitives ou langagières d'un enfant de moins de dix ans qui apprend l'espagnol... Or, je devais enseigner à de jeunes professeurs d'école ce que je ne savais pas vraiment faire moi-même... et c'est ainsi que j'ai eu l'idée à mes débuts de me mettre tout simplement à la place du jeune maître devant enseigner l'espagnol à ses élèves pour évoluer progressivement vers un dispositif de coopération action impliquant toute une école et une promotion de PE2 hispanistes, où j'ai trouvé un autre moyen de construire la formation.

\section{Trajectoire vers une métastructure de formation}

\subsection{Premier élément nouveau : faire pour comprendre}

5 Entre 1993 et 1996, j'ai donc demandé la charge de l'enseignement de l'espagnol dans deux classes de CM1 et de CM2 d'une école primaire de ZEP (Zone d'Education Prioritaire) à forte population étrangère et en particulier maghrébine, dans un quartier nommé La Paillade.

Une école d'application existait sur ce quartier, et donc un établissement dont la vocation était d'offrir un terrain d'expérimentation et de formation aux jeunes professeurs d'école de première et de seconde année ; c'est ainsi que j'ai commencé moi aussi, mes premiers cours de langue à l'école, comme n'importe quel professeur d'école débutant, avant d'entrer dans un processus qui me mènerait plus tard au système innovant proposé finalement en 2000 .

6 L'idée aurait pu paraître saugrenue à d'aucuns, puisque j'étais déjà professeur d'espagnol depuis quelques années mais, j'estimais avoir mille choses à apprendre de ces petits élèves dont je n'avais aucune habitude, avant de pouvoir conceptualiser une formation quelle qu'elle fut, pour leurs enseignants. Si je m'en tiens aux théories constructivistes de Jean Piaget ${ }^{3}(1947)$, ma plus grande difficulté était probablement que j'avais à me situer par rapport au stade de développement de l'intelligence d'un enfant d'une dizaine d'années, qui n'est pas du tout identique à celui des adultes et des adolescents plus proches mais qui s'inscrit dans un processus d'évolution allant vers une intelligence " réfléchie ». Selon Piaget, "il existerait dès le plan physiologique une «intelligence organique » laquelle se prolongerait en intelligence sensori-motrice et, en fin de compte en intelligence proprement "réfléchie » et il était très important que je situe moi enseignante débutante, le stade de mes élèves dans ce processus évolutif avant de pouvoir construire des démarches d'apprentissage adaptées à leurs capacités cognitives.

7 On trouvera d'ailleurs aussi toute une série de données plus spécifiques à l'apprentissage des langues en fonction de l'âge de l'apprenant notamment chez D. Gaonac' $h^{4}$ (1991) qui estime en reprenant de nombreux travaux, que par exemple, "alors que les enfants font environ les $2 / 3$ de leurs erreurs par rapport à la structure syntaxique des phrases et le 1/3 restant par rapport au respect de règles morphémiques, cette proportion est simplement inversée chez l'adulte"; les travaux qu'il cite concernent du reste des adultes de 18 à 25 ans et établissent très clairement que qualitativement les compétences langagières des enfants diffèrent considérablement de celles des adultes. 
8 J'ai donc ainsi dans mon ignorance, filmé seule pendant deux ans et de façon empirique n'ayant pas de formation de didactique, mes propres cours afin d'y repérer les processus, les supports, les dispositifs qui me permettraient d'avoir une action d'enseignement efficace tout en faisant que mes jeunes élèves parviennent à comprendre puis à produire de l'espagnol, tout en cherchant aussi à leur transmettre des données culturelles spécifiques du monde hispanique ; l'analyse patiente de chacune de ces vidéos après un cours, devait donc me fournir des éléments pour comprendre si mes messages avaient été entendus et comment je pouvais modifier ma démarche pour l'étape suivante.

9 J'étais donc à l'instar de mes élèves, en situation d'apprentissage et donc d'assimilation face à une réalité en mouvement puis d'accommodation; selon Piaget, "assimiler " revient à « juger » et donc à «incorporer une donnée nouvelle dans un schème antérieur ", et j'étais précisément dans cette dynamique parallèlement à mes élèves tout comme doit l'être n'importe quel enseignant débutant, je présume.

10 Il est certain qu'un double mouvement d'assimilation et d'intégration de nouvelles données et pour les élèves et pour moi-même, s'opérait en même temps que nos évolutions respectives interagissaient aussi; pour donner un exemple simple, un terme grammatical incompris des élèves appelait de ma part un recadrage sémantique moins abstrait et à l'inverse mon mécontentement ou mon refus de coopérer lors des sollicitations effusives, individuelles ou désordonnées de leur part pour obtenir de mener un mime, un jeu ou une activité finissait par modifier (pas toujours cependant...) leurs stratégies pour attirer mon attention; l'idée que l'enseignement/apprentissage fonctionne de l'enseignant vers les élèves dans une classe et vice-versa dans une double articulation me semble assez juste bien que les modalités soient un peu différentes dans un sens et dans l'autre, ne serait-ce que par l'âge, le rôle et les capacités cognitives des uns et des autres mais il est clair qu'un enseignant et ses élèves apprennent ensemble.

11 J'ai aussi à cette époque, notamment réfléchi aux exigences officielles de la circulaire sur l'EPLV (Enseignement Précoce des Langues à l'École) du 6 septembre 1991, puis de toutes celles (fort nombreuses) qui ont suivi ainsi que des divers Bulletins Officiels parus au cours de ces dix dernières années en essayant de "traduire» et de construire les demandes en termes de l'Institution en didactique de l'espagnol: cet enseignement de langue devait être basé sur une langue dite de communication, centrée sur l'enfant, ludique, essentiellement orale, active, variée, associant le «dire» et le "faire » et transmettant des notions de civilisation sur le pays dont on apprend la langue pour être brève.

12 Les méthodes faisaient donc appel à une intelligence sensori-motrice plus adaptée probablement à l'enfant, où l'assimilation était provoquée par l'expérimentation du langage via un jeu, un mime, l'utilisation du corps et des sens. J'ai donc essayé de constituer moi-même un stock d'outils, de supports pédagogiques et de techniques de classe pour l'enseignement de cet espagnol-là; essentiellement à base de jeux, de chansons, de sketches, de mimes, de travail sur la photo, la peinture, le support vidéo, la bande dessinée, avec une utilisation de la trace écrite limitée et toujours successive à un travail d'écoute, de reconnaissance, d'imitation, de reproduction puis d'investissement de notions linguistiques qui devaient pouvoir décrire ce monde autour de l'enfant de façon de plus en plus précise et riche en cercles concentriques à partir de «je» dans un mouvement d'appropriation de la langue à partir du monde connu de l'enfant ; je rappelle que dans la circulaire de 1991, l'écrit ne devait être abordé que lorsque le système phonologique était acquis, ce qui donna lieu à de nombreuses discussions passionnées où 
des enseignants de diverses langues jugeaient de cette apparition de l'écrit à un, trois ou six mois de la première leçon et avec ou sans recours à la phonétique. Tout cela était aussi soumis dans mes groupes de douze élèves à l'obligation de transmettre des notions de civilisation, c'est-à-dire selon l'Institution et ses textes, de familiariser l'enfant avec les « emblèmes nationaux » comme la monnaie (c'était avant l'euro), ou la nourriture selon la circulaire du 6 septembre 1991, mais je pris la liberté d'introduire aussi des éléments de culture patrimoniale, ce que ne demandaient pas les textes officiels car je ne pouvais m'en empêcher de faire puisque c'est une habitude très ancrée et sacrée dans l'enseignement secondaire de l'espagnol où j'avais été formée; ce phénomène a été du reste fort bien étudié par Denis Rodrigues, dans une thèse ${ }^{6}$ consacrée à l'enseignement de la civilisation hispanique en France de 1949 à 1985 ; l'espagnol en France, fait, il est vrai, parti de ces langues que l'on transmet prioritairement par le fait culturel, considérant peut-être qu'il n'est somme toute qu'une autre variation du latin, à l'instar du français et que la culture importe au-delà de tout. Je m'appliquai donc à faire apparaitre de façon aussi transversale et interdisciplinaire que possible dans mes supports autant de notions phonétiques ou linguistiques que d'éléments de civilisation centrés sur la diversité du monde hispanique.

Il m'était donc possible d'enseigner des repères spatiaux sur une carte géographique du Chili, mais aussi le lexique espagnol de la famille à partir d'une peinture de Botero représentant une famille, ou plus simplement l'heure, à partir d'un véritable emploi du temps d'un enfant catalan, ou les heures et les habitudes alimentaires dans un sketch utilisant de véritables cartes de restaurant ramenées d'un périple espagnol. Je dirai que le type d'enseignement culturel ici dispensé n'est pas sans rappeler les éléments d'approche de la culture proposés par Jean-Claude Beacco $(2000)^{7}$ dans une démarche intégrée à l'enseignement, pluraliste dans la diversité, contextuelle et donc en lien avec la réalité, active et vivante, intéressante, ludique et motivante, axée sur la communication, non généraliste (au sens de folkloriste) et non marginale, même si atteindre tous ces objectifs n'est pas chose aisée, nous le savons tous.

14 J'ai ainsi pu disposer d'un certain nombre de supports destinés à être utilisés pour des séances de 45 minutes maximum (comme le demandait l'Institution), supports centrés sur des objectifs de communication orale et permettant une alternance rapide d'activités différentes toutes les 7 à 10 minutes. La méthode d'espagnol pour le primaire, parue à peu près à l'époque de mon entrée à l'IUFM, m'a aussi énormément servi je dois dire et j'en soulignerai ici la qualité des documents, la clarté de la progression linguistique et la richesse culturelle des supports : Collection R. Basterra (septembre 1989), Mi mundo y yo, l'espagnol à l'école élémentaire - livres, niveau 1 et 2, livres du maître, cahier de l'élève et cassettes audio, Didier, Paris.

\subsection{Deuxième phase d'innovation : laisser faire et guider pour comprendre}

15 À partir de 1995, je me sentais suffisamment à l'aise, et j'avais des résultats chez mes élèves suffisamment probants à mon sens, pour me poser le problème de la formation même des futurs professeurs d'école au sein même des classes, en leur offrant des espaces pour enseigner, pour faire, puis réfléchir à ce qu'ils transmettaient et ainsi acquérir une maîtrise d'enseignant. Je créais donc un troisième niveau d'apprentissage dans la classe pour des stagiaires au fur et à mesure que je maîtrisais mieux moi-même la transmission 
de l'espagnol aux enfants et que je n'étais plus si inexpérimentée; les éléments didactiques cités précédemment restaient les mêmes pour les stagiaires en termes de contenus et de stratégies didactiques et d'attentes que je leur précisais. La caméra était restée là, les acteurs enseignants étaient devenus plus nombreux et les élèves s'en trouvaient fort satisfaits, ne sabotant jamais notre travail malgré l'importance des regards sur eux et la trace audio-visuelle que nous en gardions. Nous consacrions beaucoup d'énergie à nos préparations et à nos cours et les élèves avaient en principe, une perception spéciale, je dirai un peu sacralisée de ces enseignants pas tout à fait comme les autres qui venaient ponctuellement leur transmettre un nouveau savoir et les observaient plus qu'à l'accoutumée; ils semblaient eux aussi participer à cet intérêt nouveau en se concentrant davantage, sur ce que nous leur demandions et en attendant impatiemment ce cours un peu différent qui venait rompre les habitudes de leur semaine.

En 1996, nous avons donc repris cette expérience avec le même dispositif à l'école des Tours, école du même quartier de La Paillade, dans la classe d'un enseignant parfaitement hispanophone, Michel Ramos ${ }^{8} q u i$ a bien voulu nous accueillir après des mutations à l'école Bari faisant disparaître l'espagnol ; je saluerai sa grande patience pour nos allées et venues dans sa classe mais surtout la grande utilité de mon travail avec lui pour que je comprenne enfin l'importance de l'apport d'un enseignant en situation qui à la fois maîtrise parfaitement la langue enseignée sans être tout à fait un spécialiste et qui connaît les apprenants parfaitement révélant ainsi l'articulation entre les deux domaines; il m'a fallu cela pour comprendre enfin la construction des liens entre le savoir, l'élève et leurs contextes.

Ramos avait un net avantage sur moi : il connaissait les caractéristiques psychologiques et cognitives de ces enfants fort bien et avait de surcroit une maîtrise de la langue orale enseignée parfaite, il était plus en situation d'être médiateur que moimême et donc en situation aussi de me faire prendre conscience de cette articulation. Chaque séance effectuée par les stagiaires fut donc analysée en commun avec lui qui connaissait chaque élève de façon très précise dans sa dimension scolaire, sociale ou affective et qui pouvait aiguiller les stagiaires vers des remédiations et des évolutions tenant compte d'une globalité à laquelle je n'avais pas accès comme hispaniste et formatrice externe, n'ayant que la connaissance du savoir à transmettre. C'est à ce moment-là que j'ai pris conscience de l'importance d'une méta-structure de réflexion avec divers acteurs pour un enseignant débutant qui doit trouver un lieu où élaborer un questionnement disciplinaire et cognitif en prise avec la réalité et où construire des outils de résolution.

Ce dispositif offrait en fait un processus récursif central où des faits existants pouvaient être assimilés pour comprendre la réalité mais en même temps la réalité pouvait elle même modifier le dispositif : le hasard de la présence de Michel m'a ainsi révélé quand j'ai pris conscience de son rôle, l'importance de l'enseignant qui connaît les élèves et le contexte pour construire la formation des jeunes collègues dans la dialectique théorie pratique en lien avec un contexte.

\subsection{Laisser faire et prendre conscience par et dans une méta- structure}

19 Quasiment dix ans après mes premiers cours à la Paillade, j'ai donc abouti pendant l'année scolaire 2000-2001 à une expérience de coopération - action de formation avec 
une école primaire dans un milieu aisé puisqu'il s'agissait d'une commune de 12000 habitants assez aisée à une dizaine de kilomètres de Montpellier : Mauguio.

Cette commune présente deux aspects sociologiques et culturels caractéristiques : elle est riche d'une part, car installée sur une zone commerciale et agricole prospère, mais elle est aussi à 60 pour cent issue d'une émigration espagnole déjà très intégrée au tissu social de la ville (émigration économique des années soixante mais aussi antérieure).

Le point commun avec certaines écoles de ZEP9 ${ }^{9}$ reste cependant la valeur extrêmement positive de la langue et de la culture espagnoles dans cette ville.

Si l'identité espagnole comme partout ailleurs, n'a pas été un facteur valorisé jusqu'à la démocratisation de l'Espagne, à partir des années 1980, cette identité est devenue un véritable phénomène de mode qui dans le cas de Mauguio, se traduit par une omniprésence de l'Espagne non seulement dans la langue encore très parlée dans les familles mais dans les habitudes, dans les fêtes traditionnelles locales, où sont venus s'intégrer des célébrations espagnoles comme une composante constitutive de l'identité locale. Cette composante hispanique est une marque d'appartenance à la ville même et il est fréquent que des néo-arrivants originaires d'autres régions de France parfois très au nord, se prennent de passion pour l'apprentissage de la danse sévillane ou d'autres activités dites "espagnoles» dans un processus d'intégration à l'identité de la ville même ; il s'agit là d'une assimilation et accommodation culturelle très particulières.

J'étais donc soumise bien sûr aux aléas et réalités des classes qui nous acceptent et en l'occurrence, j'ai eu la chance d'être accueillie par Ariette Peret, enseignante de l'école Albert Camus de Mauguio, hispaniste elle-même et ravie de nous accueillir pour se prêter au jeu de constituer avec moi cette méta-structure de réflexion qui devait servir de relais aux stagiaires avant et après une séance. Le dispositif a donc consisté à demander à toute une promotion de PE2 de prendre en charge l'enseignement de l'espagnol dans les deux CM1 de l'école, après avoir élaboré la progression et des contenus sur l'année ensemble, chaque stagiaire ayant une partie de la progression à construire en doublette. Le travail des deux CM1 étant décloisonné avec Marie Julliard, la collègue d'Ariette Peret et toutes deux ont été d'accord pour ce dispositif. Chaque samedi, deux doublettes de PE2 qui préparaient ensemble une même leçon, intervenaient donc sur 2 demi classes avant et après la récréation, observant la demi doublette avec qui ils n'avaient pas préparé ; puis la fin de la matinée était consacrée à une analyse fouillée allant toujours de l'intéressé vers ses pairs puis vers nous ; avec le temps, j'ai pris un soin tout particulier à intervenir la dernière pour essayer de recenser tous les éléments mis en évidence dans chaque niveau d'analyse en essayant ensuite de chercher des pistes de réponses. Je dois avouer qu'en termes de formation, cette interactivité croisée et ce développement de stratégies méta cognitives est de loin, le dispositif qui a le plus rapidement développé des savoirs faire chez les stagiaires, à mon sens; le fonctionnement en doublettes a permis de contraster et de réunir très vite des cas très divers de problématiques et de stratégies pédagogiques pour y répondre dans un même groupe de jeunes collègues et la mutualisation de leurs questionnements, de leurs stratégies différentes et de leur travail nous a permis non seulement de constituer des ressources en termes de fiches, matériels, etc... mais aussi de poser de vrais problèmes didactiques très nombreux, très contrastés, très diversifiés avec une quantité d'informations très appréciables et de nombreuses idées de stratégies didactiques. a pu aussi servir de base de travail pour l'entrée dans le métier à la rentrée qui a suivi. 
J'étais devenue dans ce nouveau système organisatrice, facilitatrice de contenus, de démarches, relais pour l'analyse des pratiques, vecteur et dépositaire avec Ariette Peret des questionnements et des stratégies trouvées par les jeunes collègues mais plus du tout enseignante de la classe sauf exceptionnellement.

Ce genre de dispositif de formation a du reste, été semble-t-il déjà largement étudié et expérimenté pour d'autres corps de métiers où la professionnalisation est véritablement cruciale comme par exemple la médecine ${ }^{10}$; les dégâts provoqués par un médecin qui se trompe sont il est vrai, plus effrayants que les errements d'un enseignant inefficace.

On pourrait se référer notamment aux recherches de l'université de médecine Mac Master ou de Sherbrooke (Canada), de Bordeaux II qui sont impliquées dans un réseau francophone de construction pédagogique de la formation des enseignants en médecine et surtout de leurs formateurs, réseau déjà bien avancé semble-t-il.

Pour ma part, et beaucoup plus modestement, j'étais juste dans une nouvelle structure à coté du stagiaire essayant de susciter une évolution sans jamais faire à sa place.

\section{Notions sélectionnées et conservées}

25 J'ai ainsi pu constater pour commencer un phénomène que je connaissais déjà bien un peu dans le secondaire.

\section{Les impacts d'un contexte culturel et affectif sur l'apprentissage lui- même}

Dans le cas des enfants de la Paillade, un phénomène d'attrait culturel des enfants originaires du Maghreb vers l'Espagne existait tout naturellement comme pour les élèves de même origine que j'avais formés en lycée. L'Espagne située entre la France et le Maghreb, et donc intermédiaire pour de nombreux élèves, sur la route des vacances, entre deux espaces d'appartenance et de culture, était perçue comme une sorte de passerelle reliée à des expériences heureuses de vacances et cela favorisait grandement mon travail ; en effet, ces enfants avaient des $a$ priori très positifs sur ma discipline, et ce fut un atout dans des classes souvent turbulentes voire hyperactives qu'il n'était pas vraiment aisé de canaliser malgré une bonne volonté manifeste de ces enfants. L'espagnol avait pour eux un sens et ce sens était encore plus important lorsque je devais évoquer des particularismes phonétiques ou culturels légués à l'Espagne par huit siècles de présence musulmane sur le sol ibère.

27 Il était en effet, insolite et réparateur pour ces élèves d'apprendre que des Arabes (si mal perçus en France par leurs contemporains) avaient légué à l'Espagne en d'autres temps, un palais aussi magique que celui de l'Alhambra, ou des traductions précieuses que celles de tout le savoir de l'Antiquité grecque en mathématiques, médecine, astronomie, philosophie ou religion, savoir sauvé, traduit et transmis à l'Europe par ces mêmes Arabes.

Cela me faisait réfléchir plus avant à l'importance d'un contexte affectif et culturel dans tout apprentissage et cela ne pouvait pas ne pas me rappeler l'observation du même phénomène en lycée. Je citerai juste pour mémoire, le visage éclairé et ravi d'un de mes premiers élèves de seconde dont la cause me fut acquise du jour où je lui révélai que Gibraltar était étymologiquement le détroit de Tarik, son propre prénom. 
29 Je prenais ce facteur pour un détail anecdotique et amusant mais en fait, la volonté d'apprendre chez ce garçon puis chez mes jeunes élèves maghrébins de La Paillade se construisait aussi et en même temps que leur réparation culturelle et identitaire qui entrainait une motivation très forte ; cette médiation culturelle est en effet un facteur de motivation puissant et très facilitateur pour l'apprentissage et je renverrai ici aux travaux de Tolman cités par Gaonac'h (1991), en matière d'apprentissage des langues pour évoquer précisément que dans des expérimentations avec des rats mis dans des labyrinthes, la motivation par la nourriture agit de façon claire sur la performance ${ }^{11}$ physique ; bien sûr, les modalités d'apprentissage d'un jeune enfant sont infiniment plus complexes que celles d'un rat mais je pense avoir pu vérifier moi aussi, à maintes reprises le même phénomène de performance accrue par la motivation que représentait une valorisation de l'identité culturelle; il est clair que je constatais une implication plus forte de la part d'enfants maghrébins que des autres parce qu'ils se sentaient à certains moments valorisés par des contenus culturels et historiques en cours d'espagnol dans un contexte d'identité ternie et cela même si le processus d'apprentissage n'en était pas bouleversé dans ses fondements comme le souligne du reste Gaonac' $\mathrm{h} ; \mathrm{j}$ 'avais donc là une clé essentielle sur la valeur de médiation de la culture espagnole mais aussi sur la médiation culturelle par un biais historique, en cours de langue et dans un milieu culturellement conflictuel car pluriel. Nous sommes ici au cœur de la définition très claire que Geneviève Zarate (1993) donne de "l'enseignant de langue vivante comme acteur social » dans un ouvrage consacré aux représentations de l'étranger dans la didactique des langues $!^{12}$.

Utiliser le contexte pour un cours de langue deviendrait ainsi plus tard, une voie que je demanderais aux stagiaires d'explorer si possible à partir de contextes d'apprenants contrastés pour y repérer des invariants et surtout pour favoriser l'apprentissage en tenant compte de ces liens ; je ne pense pas avoir choisi du reste Mauguio au hasard pour ma dernière expérience car je souhaitais pouvoir permettre aux stagiaires de réfléchir à ce qui s'articulait entre un enfant et son contexte lors de l'apprentissage précisément dans un contexte social et affectif où l'espagnol était très signifiant. Dans le cas de Mauguio, nous étions en effet, en face d'enfants issus de catégories sociales infiniment plus aisées, globalement plus dociles, issus aussi d'une immigration espagnole déjà intégrée dans la société française depuis deux voire trois générations, mais cependant toujours très attachés affectivement à cette culture espagnole et probablement bien plus fortement encore que les enfants de La Paillade.

31 Nos stagiaires en effet, se sont souvent trouvés confrontés à de jeunes disciples très fébriles leur demandant instamment de retrouver sur la carte, tel village essentiel où ils avaient rendu visite à des parents en Espagne au cours de l'été ou tel autre où était né un grand-père espagnol; or, étant moi aussi affectivement très concernée par cette hispanité, j'ai parfois eu la faiblesse de céder, je dois l'avouer, au désarroi d'un de mes disciples devant le désir pressant d'un enfant, en indiquant moi-même un lieu ou une célébration festive, au lieu de les laisser construire leur lien d'enseignement apprentissage entre eux dans l'autonomie et dans la prise de conscience de l'impact de ce contexte sur l'apprentissage par le stagiaire.

Les cahiers de classe devenaient aussi du reste, des objets d'identité familiale où les parents d'origine espagnole observaient la progression et où les enfants collaient sans qu'on le leur demandât, des photos de leurs ascendants espagnols aux côtés des ascendants melgoriens sur les arbres généalogiques ou des photos d'eux-mêmes en 
costume traditionnel andalou en page de garde; ils s'identifiaient manifestement affectivement à cette langue et donc il m'appartenait surtout de susciter parmi les jeunes collègues ensuite, avec l'aide de l'enseignante de la classe qui connaissait aussi la famille, un questionnement sur le traitement à donner à cette motivation affective et identitaire incontournable, à cette projection dans le savoir enseigné, dans le processus d'apprentissage pour justement rendre cet apprentissage plus performant en reprenant les conclusions de Tolman.

\section{L'analyse de pratiques pour construire une problématique}

33 Je retiendrai aussi de l'ensemble du processus évoqué, qu'il est aussi absolument essentiel pour pouvoir modifier un système de pouvoir l'observer par un regard externe qui indique des voies d'évolution possibles; ce que l'on appelle communément analyse des pratiques m'est apparu peu à peu comme nécessaire pour construire une évolution dans un travail quel qu'il soit, et je sais avec certitude que plus je diversifiais les types d'examinateurs de la pratique du sujet analysé, plus le collègue formé recevait d'informations diversifiées sur sa construction de l'enseignement - apprentissage et plus il évoluait vite et de façon performante et pertinente.

Dans le système final, de coopération - action que j'ai proposé aux PE2 en formation, j'ai ainsi pu complexifier et surtout enrichir cette analyse en utilisant non seulement une caméra mais aussi plusieurs regards d'acteurs différents : le pair avec qui on a préparé son cours, celui avec qui on n'a pas préparé et qui a peut-être trouvé d'autres modalités, celui qui a observé en ayant préparé différemment sur les mêmes objectifs, l'enseignante de la classe qui a en d'autres moments abordé la même notion, puis moi, soit six niveaux d'analyse différents et complémentaires en comptant le formé lui-même.

Je dois aussi souligner que si j'ai pu mener ce travail avec succès et intégrer moi-même des données sur l'enseignement de langue en primaire au début, je le dois aussi beaucoup à l'aide, à l'acceptation et au dialogue constant qui m'a été donné par une équipe de pairs, qui se trouvait être une équipe d'enseignants extrêmement unie et efficace pour ce qui est de ma première expérience à La Paillade ; en effet, l'équipe de l'école Bari où j'ai fait mes premières armes, a travaillé au sein de cette même école une vingtaine d'années et avait une connaissance parfaite du contexte, des familles et de la façon pertinente de construire des enseignements avec ces enfants. Les enseignants ${ }^{13}$ auxquels je me réfère ici, souvent d'origine espagnole, il est vrai, et affectivement interpellés par ma discipline (on peut penser que cela les poussaient à m'aider dans ma tâche) ont été un élément fondamental de mon propre apprentissage, sans lesquels je n'aurais pas évolué aussi vite ; sans leurs informations essentielles sur les caractéristiques d'un enfant, de sa famille, d'une classe, sur les difficultés d'apprentissage, sociales, affectives en ce lieu précis, les possibles remédiations, j'aurais perdu beaucoup de temps à chercher de l'information, puis des réponses toute seule. La formation ne se limite pas, il est vrai, aux seuls moments des cours de formation et c'est une notion qui devrait être posée dans tout système qui forme; ces enseignants m'ont en particulier, permis de comprendre que certains élèves déstabilisés avaient besoin de savoir absolument si on écrivait à deux ou trois carreaux de la marge juste pour se rassurer, se poser ce que je trouvais profondément agaçant et inutile au début; ils m'ont aussi permis d'être petit à petit plus juste et exacte, plus raisonnable en termes de quantité de notions à transmettre par rapport à des élèves donnés en 45 minutes, et dans leur capacité d'assimilation. 

permis de me former mais de façon encore plus efficace avec une composante essentielle et supplémentaire : ils enseignaient tous deux ma propre discipline et connaissaient le milieu d'apprentissage tout comme mes premiers collègues de l'école Bari.

L'université de médecine Mac Master de Bordeaux que j'ai déjà citée a d'ailleurs aussi produit des publications sur des travaux de recherche sur la formation par les pairs des médecins et c'est pourquoi je soulignerai le côté fondamental du travail avec ses pairs et en situation pour la formation initiale.

D'autres phénomènes se sont aussi greffés sur mon propre apprentissage de ce milieu, il m'a ainsi fallu découvrir que ma propre réalité d'adulte, évidente à mes yeux pouvait être relative voire absurde pour les enfants, chose que je connaissais moins avec d'autres adultes ou des adolescents plus proches de moi dans leur lecture du monde que des enfants. J'avoue humblement que quelques connaissances théoriques sur l'apprentissage, sur le fait que les structures cognitives des jeunes enfants ne sont pas les mêmes que celles des adultes et que les schèmes sensori-moteurs vont progressivement être remplacés (ou complétés) par le raisonnement hypothético-déductif dans le développement de l'intelligence comme le démontre Piaget, m'auraient à cette époque beaucoup plus servi que la littérature du siècle d'Or ou l'art de la dissertation et de l'explication de texte littéraire dont j'avais été généreusement nourrie au cours de mes études universitaires.

J'ai donc eu un effort à fournir assez considérable pour comprendre l'altérité d'un enfant, pour réaliser des ajustements sémantiques, pour concevoir que la lecture de la réalité d'un enfant n'était pas calquée sur la mienne et c'est précisément ce qui m'a obligée à chercher d'autres dispositifs de formation pour les PE2, des dispositifs où justement ces décalages sémantiques pourraient être mis en relief puis assimilés et accommodés. Je l'ai notamment compris entre autres, un jour où un enfant de CM1 m'a demandé le plus sérieusement du monde si parmi les millions d'hispanophones que je venais de lui montrer fièrement sur une carte du monde, j'avais été moi-même comptée.

Je n'en savais rien... et je me sentais désarçonnée... Sa question était pertinente, logique puisque je comprenais que je n'avais aucune preuve de mes dires; peut-être voulait-il juste savoir si j'étais assez importante pour être jugée hispanophone, si j'étais une vraie locutrice, authentique et légitime à ses yeux. La réalité des chiffres que je leur donnais était elle-même subjective et je n'en avais même pas conscience, or, cela m'a poussée audelà du comique de la situation à une réflexion sur la perception de la réalité différente entre un enfant et moi et sur la nécessité de faire prendre conscience aux PE2 de ces plans de lecture différents qui doivent être un peu connus pour aider à construire des élèves.

La notion de relativité s'agissant de la représentation du monde par l'enfant est donc bien fondamentale aussi dans son apprentissage. J'ai aussi vite renoncé à cette époque, à des méthodes basées sur une réflexion métalinguistique trop fondée sur une connaissance $\mathrm{du}$ français en cours d'acquisition dans des classes en difficultés scolaires, sans toutefois écarter ces données et appauvrir l'apprentissage. Mes élèves n'avaient pas la capacité à absorber l'information comparative entre le français et l'espagnol puisqu'ils avaient quelques difficultés en français; j'ai donc évité de les noyer de façon déraisonnable. En aucun cas cela ne veut dire que je récuse le recours à une réflexion métalinguistique d'observation phonétique et écrite de deux systèmes langagiers hérités d'une même matrice : le latin; aucun élève au monde ne mérite d'être intellectuellement sous stimulé 
et $a$ fortioridans un contexte scolairement fragile; dans le cas évoqué à La Paillade, s'appuyer sur des mécanismes de réflexion métalinguistiques qui supposent une connaissance optimale du français me paraissait seulement hasardeux; la difficulté des classes où je me suis formée de façon un peu artisanale, je le reconnais, m'a seulement obligée à tenir compte des réalités de ce même contexte social, affectif et culturel pour moi-même, puis pour un dispositif de formation des PE et de façon générale, plus tard.

Si nous nous intéressons par ailleurs à tout ce que les sciences cognitives ${ }^{14}$ puis l'éthologie ont apporté ces dernières années aux savoirs sur la communication et du traitement de l'information ${ }^{15}$, nous savons que pour exister un langage doit respecter obligatoirement les trois conditions suivantes pour les éthologues notamment :

- la fonctionnalité ;

- la référentialité ;

- l'interaction sociale.

Les recherches notamment en Français Langue Étrangère ont abouti aussi à des propositions assez proches pour ce qui est des fonctions du langage mais encore plus complexes. Sophie Moirand ${ }^{16}$ (1982) répertorie quatre composantes de la compétence de communication :

- une compétence linguistique, de maîtrise du système langagier ;

- une compétence discursive, de maîtrise des registres de langue ;

- une compétence référentielle, des « domaines d'expérience et des objets du monde et de leurs relations ";

- une compétence socioculturelle, des « règles sociales et normes d'interaction entre les individus et les institutions ».

Il est assez signifiant du reste que ce qui différencie l'approche du langage par l'éthologie et par la linguistique ou des sciences plus «humaines » disons, soit précisément une insistance sur la composante discursive qui fait notre complexité même si le langage animal n'a pas encore révélé tous ses secrets et semble révéler aujourd'hui une extrême complexité en particulier pour les oiseaux. Or, c'est une nécessité que de créer un dispositif de formation qui mène le stagiaire à appréhender toutes ces données le plus richement possible et dans leur complexité.

\section{En guise de conclusion}

En définitive, l'avantage essentiel de ce type de dispositif est probablement d'offrir aux jeunes enseignants une entrée progressive dans les contenus et dans leur rapport à l'élève.

Si je me base sur les théories sur la communication de Shannon ${ }^{17}$, je dirai que la réussite qui semble avoir présidé à cette première expérience de formation / action me semble due en grande partie au découpage, à la limitation de l'information apportée à mes stagiaires. Ils ont pu se centrer sur une partie des problèmes du cours : quelques enfants, quelques aspects sans avoir à appréhender d'emblée la globalité et la complexité de savoir enseigner et sans avoir à être écrasés en termes de quantité d'information d'une classe entière. Je serais tentée de répondre qu'en termes de théorie de la communication, il est vital de sortir de cette opposition pour ne pas dire schizophrénie, car un jeune enseignant n'a ni la capacité d'absorber toute la complexité du terrain et de la décortiquer, ni celle d'ingurgiter la totalité des savoirs pédagogiques sans les relier à un vécu professionnel. 
C'est un phénomène de surdose qu'il faudrait éviter en permettant justement que la théorie soit convoquée et initiée au sein de la pratique. Peut-être devrions-nous chercher là des réponses à nos questionnements de formateurs d'enseignants; il semble qu'un balancier très récurrent existe entre théorie et pratique que l'on oppose trop dans nos métiers. Cela pose en tous cas, le problème du contexte où doit être construite la formation pour permettre une contextualisation, un apprentissage, puis une décontextualisation et un transfert de cet apprentissage vers un nouveau contexte de classe. Il a pu aussi arriver parfois que des leçons menées avec ces dispositifs semblent beaucoup plus efficaces auprès des élèves que des cours traditionnels. En effet, ayant été exposés du fait du nombre d'adultes, à plus de compréhension, ayant été davantage corrigés, stimulés par des adultes différents, les élèves ont parfois davantage progressé en expression, en compréhension, en connaissance du lexique, en maîtrise phonologique de l'espagnol. C'est une évidence. Je n'ai jamais pensé bien évidemment, que mon dispositif de formation de coopération ait été une fidèle copie de ce que ces jeunes enseignants trouveraient devant eux, seuls devant des classes à la rentrée suivante. Les élèves ont cependant bénéficié d'une quantité d'information plus adaptée, plus simple du fait d'une présence d'adultes plus importante; la pédagogie avait dans ce système plus de possibilités d'être différenciée. Quant aux stagiaires, la présence de tout leur groupe à la tête d'une seule classe a permis pour chacun d'eux de traiter moins d'aspects qu'ils n'auraient eu à en traiter s'ils avaient été seuls devant la classe et donc moins de peurs, moins d'angoisses devant une classe avec la possibilité de manier mieux une quantité de savoir plus modeste et partagée ; ils étaient moins mobilisés sur le plan de la vigilance et de la quantité d'information à traiter que s'ils avaient été seuls mais ils ont pu ainsi travailler plus sereinement et plus personnellement y compris dans la relation aux enfants qui ne reposait pas entièrement sur eux; j'étais là pour un mot oublié, une règle méconnue ou un phonème inconnu et leurs camarades aussi; la prise en charge était collective ; dans ce type de système, un stagiaire peut parfaitement construire une séance sur la numération et revenir quelque temps plus tard pour voir comment on construit une autre séance sur les repères temporels avec les mêmes élèves ; il peut aussi choisir de refaire lui-même un cours pour vérifier ses propres apprentissages d'enseignant ou les acquis des enfants et ainsi construire sa propre évolution. La seule chose qui peut m'importer en définitive, pour un enseignant débutant est qu'il reparte de sa formation avec des stratégies de réponse et non tout le savoir du monde, qu'il sache où chercher un tableau, une recette, un poème, un partenariat avec un pays hispanophone ou une règle grammaticale en termes de contenus et qu'il ait déjà un certain nombre de réflexes de savoir être et de savoir-faire au milieu de ses disciples. Je sais fort bien qu'il ne connaîtra jamais la même quantité de données qu'un spécialiste du domaine mais ce n'est pas ce qui lui est demandé. Ainsi je voudrais que celui qui voit un enfant perdu devant un arbre généalogique où la « copine de papa » n'est pas prévue, puisse lui expliquer que son lien à ses ascendants demeure le même indépendamment des trajectoires sentimentales de ses parents.

Nous avons aussi à transmettre l'autonomie à nos propres élèves enseignants et ce type de dispositif pour des stagiaires inquiets de leurs faibles connaissances disciplinaires permet un travail progressif d'appropriation sur les contenus et le rapport à l'élève.

La question de la fonctionnalité peut s'étudier fort bien aussi dans ce type de dispositif, mais en lien avec le contexte. C'est un problème complexe et les stagiaires remarquent très bien qu'ici un élève apprendra l'espagnol poussé par le désir de le parler à son grand- 
père, seul hispanophone et dépositaire d'un passé familial qui est le sien mais qui se dissout avec le temps... là, un autre souhaitera apprendre parce qu'il rêve de connaître les ruines incas et de devenir archéologue... et dans le pire des cas, les plus désintéressés s'éveilleront pour un jeu où ils voudront être meilleurs que les autres en parlant, ou même feront des efforts parce que le professeur d'espagnol leur est tout simplement sympathique... Nul n'apprend pour apprendre, faut-il le rappeler ; pas un rat ne ferait un millimètre sans une motivation ${ }^{18}$ comme un peu de nourriture... Or, dans les dispositifs évoqués, on peut assez facilement examiner quelques processus complexes et subjectifs de la motivation, du plaisir et du sens d'apprendre. En dehors d'un dispositif au sein même de l'école, un stagiaire ne pourrait pas construire une connaissance du lien entre le savoir, l'enfant et les contextes autour de cet enfant; il ne pourrait pas comprendre comment le voyage par l'Espagne d'enfants marocains de La Paillade, ou les origines, donnent du sens à l'apprentissage de la langue et c'est pourquoi un dispositif de ce genre est indiqué pour construire cette conscience des données plurielles qui interagissent lors de l'apprentissage. $S^{\prime}$ il se pouvait concevoir une formation parfaite, elle devrait selon la psychologie cognitive ${ }^{19}$ permettre un spectre d'expériences le plus riche possible mais j'ai peu parlé d'interaction sociale entre les élèves et le maitre, n'étant pas transversaliste moi-même, or, étudier des interactions aussi complexes que celles d'humains en situation d'apprentissage ne me semble pas envisageable ailleurs que dans une classe pour des enseignants en formation.

Si je n'ai pas en définitive eu l'impression d'être novatrice dans ma mission de formation, c'est probablement que l'innovation n'est rien d'autre somme toute que l'invention nécessaire dans tout processus d'acquisition et j'en reviendrai encore aux travaux de Piaget pour expliquer cette perception; selon Piaget, en effet, le sixième stade du développement de l'intelligence chez l'enfant est celui de "l'invention de moyens nouveaux par combinaison mentale $»^{20}$ et cette invention se compare à "l'application des moyens connus aux situations nouvelles ",elle "procède par déduction»,une déduction "créatrice»,qui " participe aussi des processus d'acquisition [...] et singulièrement, de la découverte des moyens nouveaux par expérimentation active "; on le voit bien le concept d'invention, de création dans l'expérimentation est un élément normal, une étape tout à fait fondamentale et incontournable dans la construction de l'intelligence sensori-motrice que Piaget appelle aussi "pratique", ne l'oublions pas, et d'une certaine manière nous formons des «praticiens » de l'enseignement même s'ils ne risquent de mettre en danger la vie de personne en se trompant de flacon.

Or, l'acquisition de la maîtrise de l'enseignement dans la classe par un jeune enseignant pourrait bien s'apparenter à l'acquisition de la maîtrise de l'intelligence sensori-motrice ou pratique chez l'enfant et sans doute à celle de la pratique du médecin.

\section{BIBLIOGRAPHIE}

ALTET M. : La formation professionnelle des enseignants : analyse des pratiques et situations pédagogiques. Paris, Presses universitaires de France, 1994. 
BEACCO J.-C. : Les dimensions culturelles des enseignements de langue. Paris, Hachette, 2000.

BERARD E. : L'approche communicative. Paris, CLE International, 1987.

BEYLOT J. : « L'enseignement de la médecine générale dans les CHU », in Projections, $\mathrm{N}^{\circ}$ 516, pp. $55-60,1991$.

COLLECTION UNIVERSITE DE TOUS LES SAVOIRS : Le cerveau, le langage, le sens, Vol. 5. Paris, Editions Odile Jacob (poche), 2002.

JANIK V. : « Whistle Matching in Wild Bottlenose Dolphins (Tursiops Truncatus) », in Science, Vol. 289 , p. $1355,2000$.

GAONAC'H D. : Théories d'apprentissage et d'acquisition d'une langue étrangère. Paris, CREDIF Hatier - Didier, 1991.

MOIRAND S. : Enseigner à communiquer en langue étrangère. Paris, Hachette, 1992.

PIAGET J. : Le langage et la pensée chez l'enfant. Lausanne (Suisse), Delachaux \& Niestlé, 1945.

PIAGET J. : La naissance de l'intelligence chez l'enfant. Lausanne (Suisse), Delachaux \& Niestlé, 1947 (ré-éd. : 1997).

PIAGET J. : Les mécanismes perceptifs chez l'enfant. Paris, P.U.F, 1961. PIAGET J. : Biologie, et connaissance. Paris, Gallimard, 1967.

PIATELLI-PALMARINI M. : Théories du langage, théories de l'apprentissage : le débat entre Jean Piaget et Noam Chomsky. Paris, Seuil, 1979.

PORCHER L. et ABDALLAH-PRETCEILLE M. : Ethique de la diversité en éducation. Paris, PUF (collection : l'éducateur), 1998.

RAPP B.C. et CARAMAZZA A. : « Disorders of Lexical Processing and the Lexicon », in The Cognitive Neurosciences. Boston (Massachusetts, USA), MIT Press, 1996.

REED S.K. : Cognition, Théories et applications. Bruxelles, (Belgique), ITP - De Bœck (collection : Université), 1999.

SHANNON C.E. et WEAVER W. : The mathematical theory of communication. Urbana (Illinois, USA), The University of Illinois Press, 1949.

ZARATE G. : Représentations de l'étranger et didactique des langues, Essais. Paris, CREDIF -Didier, 1995.

\section{NOTES}

1. Innovation : $1^{\text {er }} \mathrm{V}$. tr. Introduire dans une chose établie (qqch. de nouveau, d'encore inconnu).

V. Changer [...] V. Inventer, trouver [...]

Le petit Robert, nouvelle édition revue, corrigée et mise à jour pour 1987 p. 1006.

2. Je ne voudrais pas avoir ici à faire l'apologie de mon absence de savoir et ignorer de façon impertinente que bien des chercheurs avaient déjà expérimenté des dispositifs de formation et recherché les bases théoriques dont je manquais, mais j'ai simplement choisi d'essayer de trouver des réponses dans la pratique elle-même avant de rechercher des éléments théoriques. Je citerai seulement les travaux de Marguerite Altet qui permettent d'envisager les modalités de la formation professionnelle souhaitable pour les enseignants.

ALTET M.: La formation professionnelle des enseignants: analyse des pratiques et situations pédagogiques, Paris, Presses universitaires de France, 1994. 
3. PIAGET J. : La naissance de l'intelligence chez l'enfant. Lausanne (Suisse), Delachaux et Niestlé, 1947 (rééd. : 1997), p. 312.

4. GAONAC'H D. : Théories d'apprentissage et d'acquisition d'une langue étrangère. Paris, CREDIF Hatier - Didier, 1991, p. 141.

5. Op. cit., p. 359.

6. RODRIGUES Denis (Février 1989): L'enseignement de la civilisation hispanique en France, Discours et idéologie des manuels à l'usage du second Cycle (1949-1985). Atelier National de Reproduction des Thèses.

7. BEACCO J.-C.: Les dimensions culturelles des enseignements de langue. Paris, Hachette, 2000, pp. 132-133.

8. Michel Ramos est aujourd'hui formateur en formations transversales à l'IUFM de Montpellier, plus particulièrement en psychologie de l'enfant et il est parfaitement bilingue.

9. Zones d'Education Prioritaires: quartiers où l'on trouve des difficultés sociales et interculturelles.

10. BEYLOT J. : « L'enseignement de la médecine générale dans les CHU », in Projections, 1991, N 516, pp. 55-60.

11. GAONAC'H D. : Théories d'apprentissage et d'acquisition d'une langue étrangère. Paris, CREDIF -Hatier - Didier, 1991, pp. 70-71.

12. ZARATE G. : Représentations de l'étranger et didactique des langues, Essais. Paris, CREDIF Didier, 1995, pp. 70-71.

13. Je ne peux pas ne pas citer des collègues aujourd'hui retraités de l'école d'application Bari aujourd'hui renommée André Boulloche :

Carmen Tanchon, la directrice, Antoine Mendoza et Gilbert Carandante, enseignants ainsi que tous leurs collègues qui m'ont expliqué bien des choses sur le monde de l'école.

14. $C f$. travaux de :

ROLAND L. - de l'université d'Education de Sherbrooke (Canada) : Évaluation des apprentissages en classe, théories et pratiques - Collection : Université de tous les savoirs, Le cerveau, le langage, le sens, Vol. 5. Paris, Editions Odile Jacob (poche), 2002.

15. JANIK V. : «Whistle Matching in Wild Bottlenose Dolphins (Tursiops Truncatus) », in Science, Vol. 289, 2000, p. 1355.

RAPP B.C. et CARAMAZZA A.: "Disorders of Lexical Processing and the Lexicon", in The Cognitive Neurosciences. Boston (Massachusetts, USA), MIT Press, 1995.

16. MOIRAND S. : Enseigner à communiquer en LE. Paris, Hachette (Coll. : F), 1982, p. 20.

17. SHANNON CE. et WEAVER W. : The mathematical theory of communication. Urbana (Illinois, USA), The University of Illinois Press, 1949.

18. Cf. GAONAC'H D.: Théories d'apprentissage et d'acquisition d'une langue étrangère. Paris, CREDIF - Hatier - Didier, 1991.

19. REED S.K.: Cognition, Théories et applications. Bruxelles (Belgique), ITP - De Bœck (collection : Université), 1999.

20. PIAGET J.: La naissance de l'intelligence chez l'enfant. Lausanne (Suisse), Delachaux et Niestlé, 1947 (rééd. : 1997), Chapitre VI, p. 290. 


\section{RÉSUMÉS}

Ce travail relate mon parcours dans la formation des maîtres du premier degré à l'enseignement de l'espagnol. Il s'inscrit dans une démarche qui a consisté à construire sur plusieurs années, la formation à partir de ma propre pratique puis de celle des futurs enseignants, en recherchant progressivement des structures puis des méta structures de formation au sein de la classe et en synergie avec des enseignants expérimentés du premier degré.

This work describes my trajectory as an educational training of primary school teachers involved in the teaching of Spanish. It is part of a process which consisted of building over a period of several years, educational training based on my own practice as a teacher and then as that of future teachers, progressively developing training structures then meta-structures within the classroom and in synergy with experienced primary school teachers.

\section{INDEX}

Mots-clés : formation, invention, méta structure, pratique, réflexion

Keywords : invention, meta structure, practice, reflection, training

\section{AUTEUR}

ISABELLE ALIAGA

IUFM de Montpellier 